In der Rubrik „Literatur kompakt" werden die wichtigsten Originalarbeiten aus der internationalen Fachliteratur referiert.

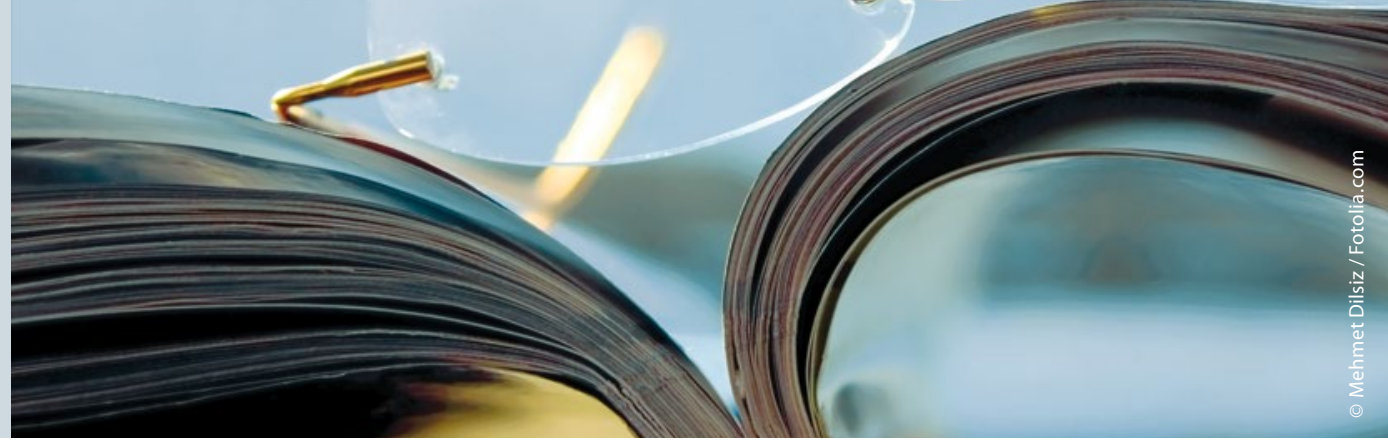

\section{To bridge or not to bridge?}

Eine Pausierung der oralen Antikoagulation steigert im Vergleich zur Überbrückung mit einem niedermolekularen Heparin nicht die Rate thromboembolischer Ereignisse, kann aber die Häufigkeit schwerer Blutungen deutlich reduzieren.

$\mathrm{D}$ as optimale Vorgehen bei Patienten, die aufgrund von Vorhofflimmern eine orale Antikoagulation (Warfarin/ Marcumar) benötigen und operiert/ interveniert werden müssen, war lange Zeit unklar. Vielerorts wurden nach dem Absetzen von Marcumar zunächst niedermolekulare Heparine bis unmittelbar vor dem Eingriff gegeben (Bridging), um das Risiko thromboembolischer Ereignisse (vor allem Apoplex) zu reduzieren. Es zeigte sich aber in der klinischen Praxis, dass diese Patienten häufig Blutungskomplikationen erlitten.

In der aktuellen US-amerikanischen BRIDGE-Studie wurde nun getestet, ob eine Heparin-Überbrückung der Mar-
cumar-Pause bei Patienten mit Vorhofflimmern im Rahmen elektiver Operationen oder invasiver Maßnahmen notwendig ist. Die Annahme der Studiengruppe war, dass eine einfache Unterbrechung der Marcumar-Therapie dem Bridging hinsichtlich der Wahrscheinlichkeit von arteriellen Thromboembolien nicht unterlegen, hinsichtlich relevanter Blutungskomplikationen jedoch überlegen ist (primäre Endpunkte).

Hierzu wurden in einer randomisierten, placebokontrollierten Studie 1.884 Patienten auf eine überbrückende Antikoagulation (Dalteparin 100 IU/kg Körpergewicht) oder Placebo randomisiert. Die bestehende Antikoagulation (War-

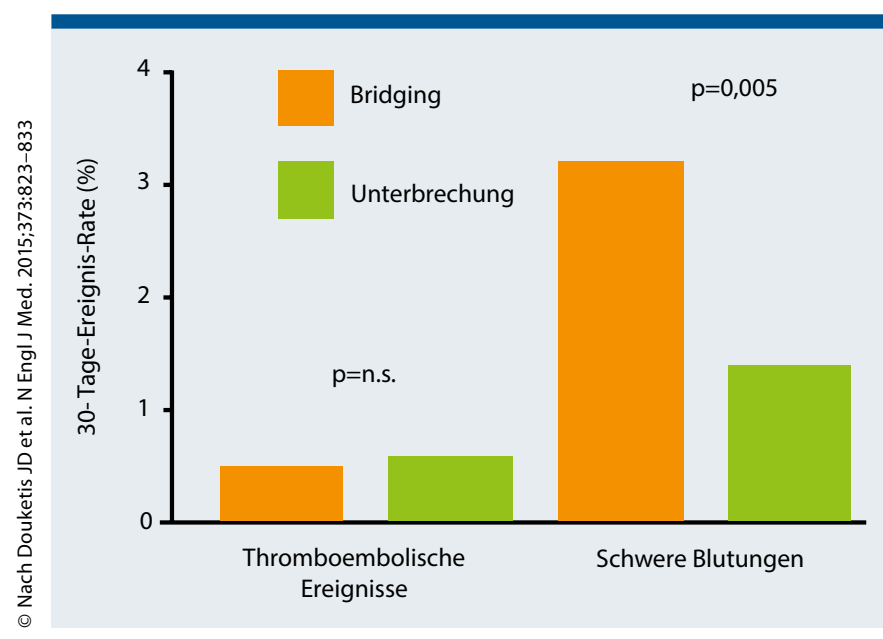

farin) wurde fünf Tage vor dem Eingriff pausiert und 24 Stunden danach wieder begonnen. Das Nachbeobachtungsintervall betrug 30 Tage.

Im Ergebnis war die Inzidenz arterieller Thromboembolien zwischen den beiden Behandlungsgruppen nahezu identisch (siehe Abbildung), wobei aber in der Bridging-Gruppe signifikant mehr schwere Blutungen auftraten als in der Placebogruppe ( 3,2 vs. $1,3 \%, p=0,005$ für Überlegenheit).

Douketis JD et al. Perioperative Bridging Anticoagulation in Patients with Atrial Fibrillation. N Engl J Med. 2015; 373(9): 823-833.

\section{Kommentar}

Diese Studie wird mit hoher Wahrscheinlichkeit Relevanz für die Leitlinien haben und das Konzept des perioperativen Heparin-Bridgings von Patienten mit Vorhofflimmern und Marcumar-Therapie mehr zur Ausnahme denn zur Regel machen. Es wird jedoch auch weiterhin Patienten mit sehr hohem Schlaganfallrisiko und vergleichsweise niedrigem Blutungsrisiko geben, bei denen weiterhin eine Überbrückung der Antikoagulationspause mit Heparin sinnvoll ist.

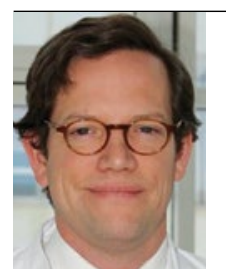

Prof. Dr. med. Peter W. Radke Klinik für Innere Medizin Kardiologie, Ereignisse und schwere Blutungen bei Patienten mit Unterbrechung der Antikoagulation gegenüber einem Bridging mit niedermolekularem Heparin. 